\title{
Representaciones sociales de la democracia en estudiantes colombianos: un análisis multidimensional y cartográfico
}

\section{Social representation of democracy in colombian students: a multidimensional and cartographic analysis}

\author{
José Ignacio Ruiz Pérez, Angélica Pineda Silva \& Ever José López
}

Universidad Nacional de Colombia

\section{Resumen:}

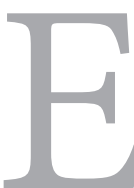

ste trabajo se propone conocer las representaciones sociales de la democracia presentes en estudiantes colombianos de carreras de educación superior, y la relación que estas guardan con las variables de victimización, pertenencia geográfica y sociodemográfica. Así mismo, se busca conocer la forma cómo se distribuye la valoración de la democracia a nivel geográfico puesto que las variables socioeconómicas y del conflicto armado inciden de forma diferencial en las regiones del país. Para ello, se cuenta con una muestra no aleatoria $(\mathrm{n}=3300)$ de carreras universitarias y técnicas-tecnológicas de 31 de los 32 departamentos del país, que respondieron un cuestionario cerrado compuesto, entre otras secciones, por una Escala de Representaciones Sociales delaDemocraciayuna Escala de Victimización. Entre los resultados se encontraron cuatro dimensiones de la democracia: valores, aspectos electorales, desconfianza y corrupción. El posicionamiento de los sujetos en estas dimensiones estuvo relacionado con la clase social y las experiencias de victimización de forma compleja. En esta vía, una proyección cartográfica muestra que no hay una relación lineal en-

\section{Abstract:}

he aim of this research was to know social representations on democracy in High school students, and relationships between this representations with victimization and geographic and demographic background variables. That, from a multidimensional and quantitative (Doise, Clémence \& Lorenze-Cioldi, 1992) view of social representations. Also, another aim was to know geographic distribution of a democracy positive-negative perception score, because of socioeconomic and war conflict variables have a differential impact in Colombian regions. To do this, a students non-randomized sample $(n=3300)$ was obtained from technological and university institutions from 31 of 32 Colombian political regions. Subjects answered a survey withaSocial RepresentationsScaleandaVictimization Scale. Results showed four democracy dimensions: Values, electoral aspects, distrust and corruption. Also, individual positions in democracy dimensions were associated in complex relations with social class and victimization experiences. Cartographic projection of a democracy perception indicator showed there is 


\section{Ciencia}

tre un indicador de valoración de la democracia con la victimización criminal.

\section{Palabras clave:}

Representaciones sociales, democracia, victimización, clase social, análisis multidimensional. not a linear relationship between this indicator and crime victimization.

\section{Key words:}

Social representations, democracy, victimization, social class, multidimensional analysis.

\section{Introducción}

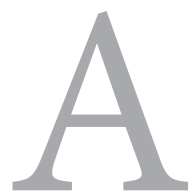

mérica Latina ha vivido recientes cambios sociales, económicos y políticos, incluyendo, entre estos últimos, conflictos armados internos y dictaduras. En esta vía, cabe mencionar el conflicto armado interno colombiano que se ha desarrollado desde la década de los cincuenta hasta la actualidad y que ha dejado un saldo de aproximadamente 220.000 muertos (GMH ¡Basta ya! Colombia; Memorias de guerra y dignidad, 2013); la guerra civil salvadoreña entre 1980 y 1992 con un saldo aproximado de 75.000 muertos (International Committee of the Red Cross, 2000); las múltiples dictaduras en Argentina, 1930 - 1932, 1943 - 1946, 1955 - 1958, 1966 - 1973, 1976 - 1983, de las cuales se estima que la última, llamada también Guerra Sucia dejo una saldo de más de 9.000 personas desaparecidas, según la Comisión Na- cional para la Investigación sobre la Desaparición de Personas (CONADEP, 1984), dato controvertido por diferentes organismos internacionales de derechos humanos que estiman que la cifra supera las 30.000 víctimas; el caso peruano en donde a lo largo del siglo XX los periodos democráticos han sido constantemente alterados por golpes de estado, siendo entonces que un periodo democrático no ha superado la decena de años y un régimen dictatorial no ha superado la docena (Flores, 1999); la dictadura militar chilena en cabeza de Augusto Pinochet entre 1973 y 1990 y que reconoce en el Segundo Informe de la Comisión Valech más de 40.000 muertos (Informe de la Comisión Presidencial Asesora para la Calificación de Detenidos Desaparecidos, Ejecutados Políticos y Víctimas de Prisión Política y Tortura, 2011). Estas situaciones, además de generar un número considerable de víctimas directas como muertos, 


\section{Ciencia}

desaparecidos o heridos, han debilitado el tejido social mediante la instauración de climas de miedo (Suárez, 1990). La aparición de regímenes políticos democráticos en estas sociedades ha sido acompañada por un auge de los estudios sobre la democracia llevados a cabo desde diferentes ópticas (Goodhart, 2007). Así, se encuentran estudios sobre la democracia en México (Echebarría \& Alvarez, 1996; Vázquez \& Campos, 2009, Yépez, s/f.), Chile (Cárdenas et al., 2007; Naranjo, 2006), o Venezuela, desde la perspectiva de las representaciones sociales y desde la óptica de la calle como un espacio de lucha ideológica (Acosta, 2009) existiendo trabajos que comparan las muestras de dos o más países. Por ejemplo, el estudio de Echebarría y Alvarez (1996) incluye una muestra del País Vasco, España, y México; y Pineda y Ruiz (2009) estudian las representaciones sociales de la democracia en muestras de cinco países: Argentina, España, Perú, El Salvador y Colombia.

En relación con este último país, existen trabajos recientes no circunscritos únicamente al ámbito de las representaciones sociales. Por ejemplo, Díaz Gómez (2006) estudió las representaciones sociales de la democracia en estudiantes universitarios mediante una encuesta estructurada de tipo abierto en torno a tres ejes principales: qué significa la democracia para el o la joven encuestado, cómo se vive la democracia en Manizales y cuáles son las condiciones que requieren los jóvenes para vivir en democracia. Entre otros resultados, el autor encontró que las respuestas a la pregunta sobre el significado de la democracia podían agruparse en bloques temáticos como «la acción de lo político», «el sistema adaptativo», «el espacio para el ejercicio de la individualidad y autonomía», «el poder del pueblo» y «los derechos y deberes». Por su parte, Ruiz y Coy (2004) estudiaron las representaciones sociales de la democracia en estudiantes de tres carreras y tres tipos de universidades (pública, privada laica y privada confesional) por medio de una tarea de asociación libre de palabras, combinada con una técnica adaptada de Guimelli (1996) sobre los esquemas cognitivos de base (ECB); hallando que el nodo central de la democracia comprendía la libertad, la participación y la igualdad, y que el concepto de democracia ocupaba un lugar central (luego, más importante, en la lógica de los ECB) para los sujetos de estratos socioeconómicos medios y bajos que para los de estratos altos.

En la misma línea, otro estudio (Rodríguez y Seligson, 2009) aborda la cultura democrática en la población colombiana. Este estudio conforma el sexto de una serie iniciada en el 2004, sobre diferentes aspectos de la opinión pública hacia el sistema democrático. Hay que señalar que las versiones del 2006 y 2008 incluyen datos de otros países del continente americano, constituyendo así uno de los pocos estudios de amplitud transnacional, a lo que se une el mérito de basarse en encuestas cara a cara de muestras de todas las regiones del país, con lo que se evita el sesgo impuesto por las encuestas telefónicas o las 


\section{Ciencia}

centradas en las ciudades más grandes del país. Entre otros resultados, en el caso colombiano, se identificó una aceptación alta hacia la democracia como sistema político y hacia diferentes formas de participación política, aunque los encuestados se manifestaron más intolerantes frente a los derechos de la oposición y de las minorías que los ciudadanos de otros países; y perciben además un nivel promedio de la legitimidad de las instituciones políticas nacionales (sin contar la percepción sobre el Gobierno Nacional vigente en la época en que se aplicó la encuesta).

Por otro lado, la apertura a nuevos sistemas políticos democráticos en varias sociedades latinoamericanas ha surgido a la par con un incremento de las tasas de criminalidad, la aparición de modelos económicos neoliberales en el marco de estructuración en Estados débiles y, relativamente, altos niveles de percepción de inseguridad (Naredo, 2001; Ruiz \& Turcios, 2009). Así mismo, se han identificado algunos factores sociopolíticos que amenazan la consolidación de la democracia en los países latinoamericanos; por ejemplo, la tensión entre instituciones militares y político-democráticas en contextos de reciente guerra civil (Schultze-Kraft, 2005), el acceso diferencial a la protección del sistema judicial y la eficacia de la ley en la persecución del crimen (Méndez, O’Donnell \& Pinheiro, 2009). Dentro de la misma línea, volviendo al estudio de Rodríguez y Seligson (2009) en el caso colombiano, se advirtió que las personas víctimas de la corrup- ción perciben una menor legitimidad en dichas instituciones (frente a las no víctimas); pero una mayor corrupción, o el hecho de haber sido víctima de algún delito se asoció con un mayor apoyo a la democracia en sí misma y con una mayor tolerancia política.

Este tipo de resultados muestran que la democracia en América Latina puede verse afectada o reforzada por diferentes factores. Además de estos, en Colombia parecen incidir otra serie de factores atípicos en la estabilidad y fortaleza de las instituciones democráticas. De este modo, primero, se ha considerado a Colombia como uno de los países con mayor estabilidad democrática de su entorno, pero al mismo tiempo, una gran parte de esas etapas de democracia formal han coexistido con la vigencia de estados de excepción que han limitado el ejercicio de derechos constitucionales (Amnistía Internacional, 1994). Segundo, desde el punto de vista del sistema electoral, la participación en las votaciones ha sido tradicionalmente baja (Hernández, 1986), estando a menudo por debajo del 50\% del electorado. Tercero, con la extensión del conflicto armado y el auge de los grupos armados ilegales, la baja participación electoral como tendencia general subsume dos fenómenos opuestos: una muy baja tasa de votación en regiones controladas por grupos guerrilleros que, bajo la modalidad de paros armados, buscaban sabotear los procesos electorales; y, en el extremo opuesto, tasas de participación muy altas en regiones en las que los grupos paramilitares 


\section{Ciencia}

pretendían que salieran elegidos los candidatos apoyados por ellos (Flórez, 2007).

El presente estudio de análisis cartográfico apunta a concluir que las representaciones sociales de la democracia pueden ser distintas en las regiones del país, si se tienen en cuenta las diferencias históricas de presencia y exposición de las poblaciones a las vicisitudes de la guerra. Así, el objetivo general de este trabajo es: conocer las representaciones sociales de la democracia en estudiantes de carreras universitarias, técnicas y tecnológicas de las capitales de los departamentos. De manera complementaria, se pretende analizar las relaciones que existen entre estas representaciones sociales con variables como la victimización criminal, dada la discrepancia de resultados que muestran los estudios. Por un lado, la relación entre una mayor experiencia de victimización y una mayor identificación con la democracia que mostraba el estudio de Rodríguez y Seligson (2009) y, por otro, la hipótesis de que más experiencias de victimización y mayor miedo al delito podrían generar una disminución del apoyo a los sistemas democráticos (Villarreal \& Silva, 2006).

\section{Metodología}

Participantes. La muestra estuvo conformada por 3300 sujetos, el $49.8 \%$ de carreras técnicas o tecnológicas con una predominación de la participación de miembros de administración de empresas; y el 50.2\% de carreras universitarias, sobre todo de programas de psicología. Las edades oscilaron entre los 16 y los 60 años, si bien el 90\% de los sujetos indicaron tener hasta 27 años de edad (media 21,61 y desviación típica: 4.36). El hecho de $10 \%$ de encuestados tienen edades superiores a los 27 años, y un límite de 60 años se debe a que el SENA ofrece capacitaciones sin discriminación de edad dentro de una oferta muy amplia que posibilita el reciclaje profesional o los convenios con otras instituciones para favorecer la inclusión laboral de adultos. Por otro lado, casi dos tercios de los entrevistados fueron mujeres (63\%), y el 86\% de los participantes eran solteros. En cuanto al nivel socioeconómico, se contó con un predominio de estratos bajos (estratos 1 y 2 en la clasificación colombiana constituyeron el $57.1 \%$ de la muestra), seguido de las clases medias (estratos 3 y 4, con $35.7 \%$ de los sujetos), mientras que las clases altas (estratos $5 \mathrm{y}$ 6) representaron el 7,3\% del total de encuestados. Finalmente, hay que indicar que la muestra proviene de 31 de los 32 departamentos del país (las muestras que no se pudieron recoger al momento de elaborar este artículo corresponden al departamento del Casanare).

Instrumentos. Los estudiantes respondieron un cuestionario compuesto en su mayoría por es- 


\section{Ciencia}

calas cerradas, de las cuales destacamos para el presente artículo las siguientes:

\section{- Escala de Representaciones Sociales de la} Democracia: es una escala que consta de 20 ítems en formato Likert, correspondiente a una perspectiva cuantitativa de las representaciones sociales (e.g., Doise, Clémence \& Lorenzi-Cioldi, 1992). Esta escala se extrajo de los resultados de una investigación previa (Pineda \& Ruiz, 2009) en la cual los datos textuales obtenidos mediante una tarea de asociación de palabras, combinada con una adaptación del modelo de los Esquemas Cognitivos de Base y analizados mediante análisis de correspondencias múltiples, arrojaron una serie de campos semánticos que sirvieron de base para elaborar los ítems de la escala.

- Escala de Victimización: contiene 15 sucesos que, analiza episodios que en los seis meses anteriores a responder la escala le pudieron haberle ocurrido al encuestado (robo, amenazas, secuestro, agresión sexual, extorsión, acoso en el lugar de trabajo o de estudio, lesiones por accidente vial, ser perseguido en la calle, recibir llamadas obscenas de desconocidos, haberle exigido dinero por un trámite público gratuito) o a un allegado familiar o amigo (lesiones por un accidente vial, muerte por un accidente vial, muerte violenta, desaparición forzada).
- Datossociodemográficos:edad,sexo,estrato socioeconómico del lugar de residencia, carrera estudiada, y estado civil.

Procedimiento. Se parte de un diseño exploratorio, sin manipulación de variables, con una muestra no aleatoria compuesta por estudiantes universitarios y carreras técnicas o tecnológicas del Servicio Nacional de Aprendizaje (SENA), que tiene sedes y programas académicos en prácticamente todas las capitales de los departamentos de Colombia. En el caso de las universidades, al no existir ninguna institución con implantación en todo el territorio nacional, se realizaron los contactos correspondientes con diferentes instituciones con el fin de contar con la respectiva muestra en cada departamento, dando preferencia a los estudiantes de psicología. En el caso del SENA, se buscó privilegiar la obtención de la muestra de estudiantes de administración de empresas. Los datos fueron recogidos durante el segundo semestre del año 2010, a través de aplicaciones colectivas en las cuales se informó previamente a los sujetos sobre el objetivo general de la encuesta y el carácter voluntario y anónimo en su implementación.

\section{Resultados}

En primer lugar se calculó la media y la desviación típica de los ítems de la escala, de acuerdo con el procedimiento sugerido por Doise, Clémence \& Lorenzi-Cioldi (1992) para identificar el núcleo central de la representación social a partir 


\section{Ciencia}

de las medias más altas. La Tabla 1 recoge estos estadísticos permitiendo apreciar que, de acuerdo con los promedios más altos, la democracia se representa contaminada seriamente por la corrupción en la elección de cargos, especialmente, del dirigente que se necesita para administrar la nación, y cooperar entre todos para el desarrollo presidido por valores como la solidaridad, la honestidad y la tolerancia. Llama la atención que los elementos nucleares de la representación social encontrados en una investigación precedente (Pi- neda \& Ruiz, 2009) como la igualdad, la justicia y la libertad, reunidos en el ítem 12 no presentan una media especialmente alta.

Por otro lado, las medias más bajas, vale decir, los aspectos de la democracia con los que menos se identifican los sujetos encuestados se relacionan con la desconfianza en los esfuerzos políticos, lo cual indicaría cierto pesimismo. No obstante, no se percibe la democracia como una forma de mantener condiciones de desigualdad, o como un estado social imposible de alcanzar.

Tabla 1.

Estadísticas descriptivas en la Escala de Representaciones Sociales de la Democracia.

\begin{tabular}{lcc}
\multicolumn{1}{c}{ ITEM } & M & D \\
\hline Es un sistema de elección de los cargos de un gobierno mediante el voto. & 2.94 & 1.00 \\
\hline $\begin{array}{l}\text { Es un conjunto de deberes y derechos que se traducen en leyes derivadas de la } \\
\text { Constitución. }\end{array}$ & 2.86 & .86 \\
\hline Es un sistema de valores para organizar el futuro de la sociedad. & 2.85 & .89 \\
\hline Es una forma de pensamiento. & 2.77 & .94 \\
\hline Es una manera para que las mayorías alcancen el poder político. & 2.62 & 1.00 \\
\hline $\begin{array}{l}\text { Es una forma de lograr el consenso para que la sociedad tenga un presidente } \\
\text { o líder. }\end{array}$ & 2.95 & .89 \\
\hline Es tener esperanza en los resultados de los esfuerzos políticos. & 2.48 & .92 \\
\hline $\begin{array}{l}\text { Es una manera de proporcionar armonía, bienestar y tranquilidad a la socie- } \\
\text { dad. }\end{array}$ & 2.66 & .94 \\
\hline Es garantizar la autonomía y la unidad en el desarrollo de la sociedad. & 2.74 & .92 \\
\hline $\begin{array}{l}\text { Está afectada gravemente por la corrupción. } \\
\text { Es cooperar desde el dialogo, la solidaridad, la honestidad y la tolerancia, para }\end{array}$ & 3.21 & .99 \\
\hline lograr oportunidades de desarrollo. & 2.90 & .91 \\
\hline Se relaciona sobretodo con la igualdad, la justicia y la libertad. & 2.88 & .96 \\
\hline
\end{tabular}




\section{Ciencia}

\begin{tabular}{lcc}
\hline \multicolumn{1}{c}{ ITEM } & M & D \\
\hline $\begin{array}{l}\text { Es una forma de compromiso sobre la equidad, fundamentado en la confian- } \\
\text { za, la lealtad y la responsabilidad. }\end{array}$ & 2.81 & .91 \\
\hline Es una estrategia para mantener la desigualdad, la injusticia y la pobreza. & 2.38 & 1.03 \\
\hline $\begin{array}{l}\text { Es la forma como se pueden llevar a cabo cambios y decisiones, de una forma } \\
\text { organizada. }\end{array}$ & 2.85 & .87 \\
\hline Realmente no existe, es una mentira y una utopía. & 2.37 & 1.01 \\
\hline $\begin{array}{l}\text { Es ante todo, un mecanismo de derechos y deberes para tener representación } \\
\text { en el congreso. }\end{array}$ & 2.64 & .85 \\
\hline $\begin{array}{l}\text { Es tener libertad de expresión y ser comprendido por los que piensan dife- } \\
\text { rente. }\end{array}$ & 2.79 & .93 \\
\hline Es un sistema de control de la economía de una nación. & 2.61 & .89 \\
\hline $\begin{array}{l}\text { Permite la expresión de la diversidad de la ciudadanía al participar en la elec- } \\
\text { ción de autoridades. }\end{array}$ & 2.86 & .93 \\
\hline
\end{tabular}

En segundo lugar, se buscó identificar los principios organizadores de la representación social para lo cual se recurrió al análisis de los factoressubyacentesalaEscaladeRepresentaciones Sociales dela Democracia.Para ello secalculó como paso previo el coeficiente KMO y el del test de esfericidad de Barlett. El primero fue de 0,925 , lo cual es muy satisfactorio, mientras que el segundo arroja un valor de Chi2 de 26789, con p <.001 y 190 grados de libertad. Los valores de ambos coeficientes indican que las correlaciones subyacentes a la matriz de ítems son suficientemente altas para proceder al análisis factorial (Martín, Cabero \& de Paz, 2007).

Los resultados del procedimiento, con rotación varimax se muestran en la Tabla 2 , señalando una solución factorial de cuatro factores.
La primera dimensión o Factor 1 a la luz de los pesos factoriales más altos, podría denominarse «los valores de la democracia», puesto que reúne los ítems sobre igualdad, justicia, libertad, equidad, confianza, lealtad y responsabilidad, autonomía y libertad. El Factor 2, agrupa varios aspectos asociados con la elección de cargos y la democracia como un mecanismo establecido por la Constitución. Por ello, se puede designar a este factor como «elecciones como mecanismo constitucional». El Factor 3, refleja una «desconfianza en la democracia», como algo inalcanzable o que puede ser empleado para mantener condiciones de desigualdad mediante el control de la economía en concordancia con los tres ítems con mayor carga factorial, que saturan dicho eje. Por último, el Factor 4 es básicamente monoítem, acerca de la «corrupción». 


\section{Ciencia}

Tabla 2.

Análisis de componentes principales con rotación varimax de la escala de representaciones sociales de la democracia.

\begin{tabular}{|c|c|c|c|c|}
\hline ITE & $\begin{array}{l}\text { Factor } 1 \\
\text { Valores }\end{array}$ & $\begin{array}{c}\text { Factor } 2 \\
\text { Elecciones }\end{array}$ & $\begin{array}{l}\text { Factor } 3 \\
\text { Desconf. }\end{array}$ & Factor 4 \\
\hline
\end{tabular}

Se relaciona sobre todo con la igualdad, la justicia y la libertad.

Es una forma de compromiso sobre la equidad, fundamentado en la confianza, la lealtad y la .802 responsabilidad.

Es cooperar desde el diálogo, la solidaridad, la honestidad y la tolerancia, para lograr oportunidades de desarrollo.

Consiste en garantizar la autonomía y la unidad en el desarrollo de la sociedad.

Es una manera de proporcionar armonía, bienestar y tranquilidad a la sociedad.

Es la forma en que se pueden llevar a cabo cambios y decisiones, de una forma organizada. .673

Es tener libertad de expresión y ser comprendido por los que piensan diferente.

Permite la expresión de la diversidad de la ciudadanía al participar en la elección de autoridades.

Es un sistema de elección de los cargos de un gobierno mediante el voto.

Es una forma de lograr el consenso para que la sociedad tenga un presidente o líder.

.667

Es un conjunto de deberes y derechos que se traducen en leyes derivadas de la Constitución.

Es una manera de que las mayorías alcancen el poder político. 


\section{Ciencia}

\begin{tabular}{|c|c|c|c|c|}
\hline ITEM & $\begin{array}{l}\text { Factor } 1 \\
\text { Valores }\end{array}$ & $\begin{array}{c}\text { Factor } 2 \\
\text { Elecciones }\end{array}$ & $\begin{array}{l}\text { Factor } 3 \\
\text { Desconf. }\end{array}$ & $\begin{array}{c}\text { Factor } 4 \\
\text { Corrupción }\end{array}$ \\
\hline $\begin{array}{l}\text { Es un sistema de valores para organizar el futu- } \\
\text { ro de la sociedad. }\end{array}$ & .498 & .516 & & \\
\hline Es una forma de pensamiento. & & .488 & & \\
\hline $\begin{array}{l}\text { Es tener esperanza en los resultados de los es- } \\
\text { fuerzos políticos. }\end{array}$ & .389 & .487 & & -.366 \\
\hline $\begin{array}{l}\text { Es una estrategia para mantener la desigual- } \\
\text { dad, la injusticia y la pobreza. }\end{array}$ & & & .771 & \\
\hline $\begin{array}{l}\text { Realmente no existe, es una mentira y una uto- } \\
\text { pía. }\end{array}$ & & & .728 & \\
\hline $\begin{array}{l}\text { Es un sistema de control de la economía de una } \\
\text { nación. }\end{array}$ & & & .603 & \\
\hline $\begin{array}{l}\text { Es ante todo, un mecanismo de derechos y de- } \\
\text { beres para tener representación en el congreso. }\end{array}$ & & .376 & .402 & \\
\hline Está afectada gravemente por la corrupción. & & & & .787 \\
\hline Autovalor. & 5.29 & 3.14 & 1.840 & 1.12 \\
\hline \% Varianza. & 26.45 & 15.72 & 9.210 & 5.61 \\
\hline
\end{tabular}

A continuación, se exploró las tipologías de sujetos que podían presentarse en las dimensiones factoriales halladas en el análisis precedente. Para ello, se realizó un análisis de clasificación jerárquica con distancia eucludiana y método de Ward, sobre las puntuaciones factoriales. Estos parámetros se recomiendan para el análisis de clasificación jerárquica aplicado a las representaciones sociales (Doise, Clemence. \& Lorenzi-Cioldi, 1992; Doise, Spini \& Clémence, 1999; Echebarría \& González, 1995). De las soluciones de clusters, se optó por la de cuatro grupos, con ella se realizó un análisis de varianza de una vía con las dimensiones factoriales para identificar la dirección de las diferencias entre la tipología de sujetos escogida. Estos resultados se muestran en la Tabla 3, en la cual se incluye además el cruce mediante la prueba Chi2 entre dicha tipología y las variables sociodemográficas. Para el estrato, se recodificó la variable, agrupando los valores en tres clases: baja (estratos 1 y 2), media (estratos 3 y 4) y alta (estratos 5 y 6 ). 


\section{Ciencia}

Tabla 3.

Tipologíasdesujetosenlasdimensioneslasrepresentacionessocialessobrelademocracia.Comparación de medias en las dimensiones factoriales y prueba de Chi2 en los datos sociodemográficos (análisis de varianza para edad).

\begin{tabular}{|c|c|c|c|c|c|}
\hline $\begin{array}{l}\text { Dimensiones de la escala de RS de } \\
\text { la democracia }\end{array}$ & $\begin{array}{l}\text { Grupo } 1 \\
(\mathrm{n}=908)\end{array}$ & $\begin{array}{l}\text { Grupo } 2 \\
(\mathrm{n}=587)\end{array}$ & $\begin{array}{l}\text { Grupo } 3 \\
(\mathrm{n}=815)\end{array}$ & $\begin{array}{l}\text { Grupo } 4 \\
(\mathrm{n}=995)\end{array}$ & $\begin{array}{l}F(3.3301)= \\
\quad L \&=\end{array}$ \\
\hline Valores & $\begin{array}{l}.501 \\
(.778)\end{array}$ & $\begin{array}{l}.659 \\
(.701)\end{array}$ & $\begin{aligned}-0.737 \\
(1.049)\end{aligned}$ & $\begin{array}{l}-.243 \\
(.767)\end{array}$ & $\begin{array}{l}467.5^{\star * *} \\
61.12^{\star * *}\end{array}$ \\
\hline Elecciones & $\begin{array}{l}.955 \\
(.653)\end{array}$ & $\begin{array}{l}-.273 \\
(.670)\end{array}$ & $\begin{array}{l}-.066 \\
(.948)\end{array}$ & $\begin{array}{l}-.656 \\
(.789)\end{array}$ & $\begin{array}{l}716.4^{* * *} \\
47.39^{* * *}\end{array}$ \\
\hline Desconfianza & $\begin{array}{c}.037 \\
(1.119)\end{array}$ & $\begin{array}{l}-.918 \\
(.631)\end{array}$ & $\begin{array}{l}.536 \\
(.846)\end{array}$ & $\begin{array}{l}.068 \\
(.786)\end{array}$ & $\begin{array}{l}315.2^{\star * *} \\
92.54^{\star * *}\end{array}$ \\
\hline Corrupción & $\begin{array}{l}-.345 \\
(.775) \\
\end{array}$ & $\begin{array}{c}.740 \\
(.669) \\
\end{array}$ & $\begin{array}{c}.814 \\
(.656) \\
\end{array}$ & $\begin{array}{l}-.789 \\
(.748) \\
\end{array}$ & $\begin{array}{c}1019.8^{* * *} \\
8.31^{\star * *}\end{array}$ \\
\hline Datos sociodemográficos & & & & & Chi2 \\
\hline $\begin{array}{l}\text { Edad: \% sujetos por encima de la } \\
\text { mediana }(\mathrm{me}=21.0)\end{array}$ & $39.5 \%$ & $32.93 \%$ & $38.34 \%$ & $35.92 \%$ & $7.58+$ \\
\hline Sexo (\% hombres) & $37.4 \%$ & $29.9 \%$ & $35.1 \%$ & $42.6 \%$ & $27.08^{* * *}$ \\
\hline Estado Civil (\% solteros) & $84.7 \%$ & $90.4 \%$ & $82.9 \%$ & $87.2 \%$ & $32.29^{* * *}$ \\
\hline Estrato Clase Baja & $61.8 \%$ & $54.7 \%$ & $47.8 \%$ & $61.9 \%$ & \\
\hline Clase Media & $32.7 \%$ & $37.8 \%$ & $41.2 \%$ & $32.4 \%$ & $56.03^{* * *}$ \\
\hline Clase Alta & $5.4 \%$ & $7.4 \%$ & $11.0 \%$ & $5.6 \%$ & \\
\hline $\begin{array}{l}\text { Tipo de carrera } \\
\text { (\% con carrera universitaria) }\end{array}$ & $47.6 \%$ & $60.0 \%$ & $59.1 \%$ & $38.1 \%$ & $109.08^{* * *}$ \\
\hline
\end{tabular}

\& F: Análisis de varianza, $\mathrm{L}=$ Prueba de Levene de homogeneidad de varianzas.

$+\mathrm{p}<.10 ;{ }^{* * *} \mathrm{p}<.001$

De acuerdo con este análisis, el primer grupo se caracteriza, en primer lugar, por una concepción de la democracia ligada a los mecanismos formales electorales y en segundo lugar, ligada a los valores subyacentes a la democracia sin considerar que esté afectada por la corrupción. Predominan en este grupo, los sujetos de estrato bajo y de carreras técnicas o tecnológicas (\% complementario de 


\section{Ciencia}

la tasa de sujetos con carreras universitarias). Por ello, denominaremos a este grupo como «Electoralistas hacia los valores democráticos».

El segundo grupo, puntúa especialmente alto en la dimensión de los valores de la democracia, y aunque también consideran que está permeada gravemente por la corrupción, los encuestados no creen que la democracia sea una estrategia de dominación ni que favorezca desigualdades. En este grupo, la presencia de mujeres, de solteros, de sujetos de clase media o alta y con carrera universitaria es mayor. De acuerdo con este perfil, denominaremos a este grupo como «Idealistas democráticos preocupados por la corrupción».

Por otra parte, el tercer grupo se caracteriza por una visión pesimista de la democracia pues muestra puntuaciones altas en la dimensión de desconfianza y de corrupción, bajas en los valores y en los aspectos que rodean las elecciones. Predominan aquí los sujetos de clase media o alta y con formación universitaria, a los cuales designaremos como «Pesimistas ilustrados». Por último, el cuarto grupo presenta puntajes bajos en las cuatro dimensiones de la democracia, especialmente en la de corrupción, por lo que podríamos definir a este grupo como «Desapegados hacia la democracia».

Teniendo en cuenta, a nivel de medias, el puntaje que alcanzan los ítems y la estructura factorial de la escala (ver Tabla 3) con dos factores que reúnen aspectos positivos de la democracia, y otros dos centrados en aspectos negativos o problemáti- cos, se procedió a calcular un Índice de Valoración de la Democracia (IVD). Para ello, se sumaron los puntajes de los ítems positivos de la democracia (Factores 1 y 2) y se dividió el resultado por el número de ítems. Por otra parte, con los ítems que se refieren a los aspectos negativos de la democracia se procedió de la misma manera: se sumaron los puntajes directos y se dividió el resultado entre el número total de ítems. Así, se obtuvieron dos coeficientes: uno sobre aspectos positivos de la democracia y el otro sobre aspectos negativos. La resta del segundo sobre el primero arrojó el IVD, un nuevo coeficiente cuyos puntajes a nivel de los sujetos podría oscilar entre +3 (representación social totalmente positiva de la democracia) y -3 (representación negativa). La distribución de puntuaciones en esta nueva variable se aproxima a la de una curva normal, con una media algo positiva (0.45, $\mathrm{DT}=0.96)$, muy próxima a 0 . En otras palabras, no se encuentra una clara prevalencia de los aspectos positivos de la democracia en la representación que de ella tienen los sujetos encuestados.

Posteriormente, se exploró si había alguna relación entre la victimización sufrida y la posición que se tiene sobre el concepto de la democracia en cuanto a la adscripción a alguno de los grupos identificados anteriormente. Para ello, se realizó un análisis de los componentes principales y la rotación varimax de los sucesos de la Escala de Victimización Criminal. Esto arrojó tres factores: el primero se denominó Daños físicos, debido a que se saturó de la manifestación de ítems 


\section{Ciencia}

como "muerte en accidente de familiar o amigo/a» (766), de «lesiones en accidente de familiar o amigo/a» (745), «muerte violenta de familiar o amigo/a» (.689) y «desaparición forzada» (421). El segundo factor se denominó Delitos coactivos pues agrupó la presentación de ítems como «secuestro» (789), «agresión sexual» (.714), «extorsión» (600) y «acoso en lugar de trabajo/estudio». $\mathrm{Y}$ el tercer factor recogió ítems como «amenazas» (649), «persecución en calle» (629), «robo/hurto» (551), «agresión física» (516), «llamadas obscenas» (429) y «dinero por trámites públicos gratuitos» (375). El conjunto de los tres factores explicaban el 39.96\% de la varianza, 14.21\%, $12.97 \%$ y $12.78 \%$ respectivamente por cada factor.

A partir de ello, se calcularon las correlaciones bivariadas entre: los factores de victimización y un indicador total de victimización (sumando los puntajes brutos en cada ítem de la escala de victimización) y estas con las tipologías extraídas del análisis de conglomerados y con el Indicador de Valoración de la Democracia. Los resultados se exponen en la Tabla 4. Cada clase de la tipolo- gía de cuatro clusters analizada anteriormente se transformó en una variable dicotómica para poder realizar el análisis entre esa clase y los indicadores de victimización: por ejemplo, para la clase «Electoralistas hacia los valores democráticos» se asignó un 1 al sujeto si había quedado agrupado en esa categoría, y 0 en caso contrario.

De acuerdo con lo hallado, la relación entre victimización y RS de la democracia es compleja. Por un lado, contar con una mayor experiencia como víctima de daños físicos (accidentalidad vial, muertes violentas y desapariciones) se asoció con una valoración más positiva de la democracia (IVD) y caracterizó más a los sujetos cuya RS de la democracia fue más «Electoralista». Por otro lado, los sujetos correspondientes al grupo «Idealista» se caracterizaron por presentar menores experiencias de delitos en general y de delitos en la calle en particular. En cambio, los sujetos más «desapegados» de la democracia indicaron haber sufrido un mayor número de experiencias de daños coactivos, delitos en la calle y más delitos en general.

Tabla 4. Correlaciones bivariadas de Pearson entre indicadores de victimización y de democracia.

\begin{tabular}{lccccc} 
Victimización & Electoralistas & Idealistas & Pesimistas & Desapegados & IVD \\
\hline Daños físicos & $.04^{*}$ & -.19 & .004 & -.032 & $.59^{* *}$ \\
\hline Daños coactivos & .003 & -.032 & -.018 & $.041^{*}$ & -.20 \\
\hline Delincuencia en calle & -.028 & $-.069^{* * *}$ & .020 & $.067^{* * *}$ & -.11 \\
\hline Total victimización & .010 & $-.070^{* * *}$ & .012 & $.038^{*}$ & .24 \\
\hline
\end{tabular}

${ }^{*} \mathrm{p}<05 ;{ }^{* *} \mathrm{p}<01 ;{ }^{* *} \mathrm{p}<001$ 


\section{Ciencia}

Figura 1.

Distribución geográfica de la Valoración de la Democracia y de un Indicador de Delincuencia
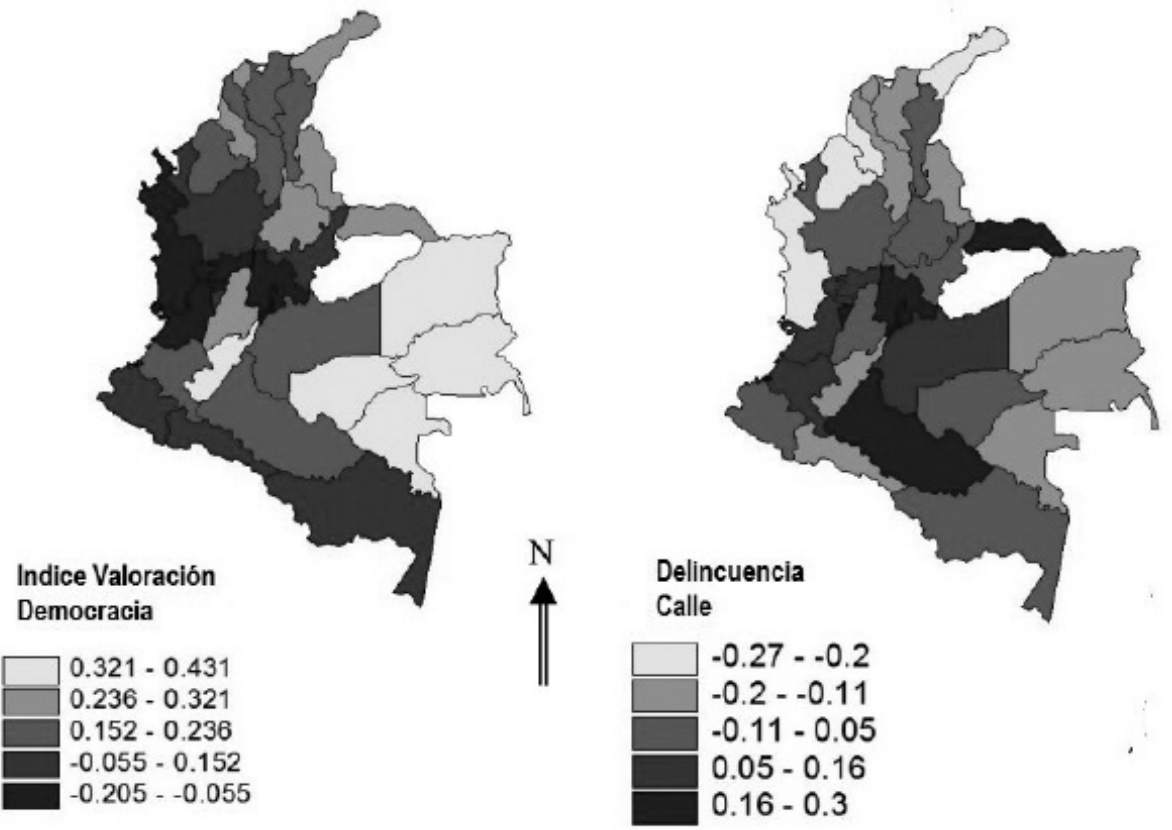

Por último, se analizaron las posibles diferencias entre las regiones del país, tomando como unidad geográfica el departamento y su relación con indicadores de criminalidad. En la Figura 1, se muestra la distribución geográfica del Índice de Valoración de la Democracia, donde las zonas más oscuras indican una mayor valoración de los aspectos negativos. Al lado del mapa, se muestra la distribución de un indicador de delincuencia obtenido en un análisis factorial (Ruiz, en revisión) que agrupa las situaciones de persecución en la calle por desconocidos, amenazas, robos/

hurtos, agresión física, llamadas obscenas y haber pagado por trámites públicos gratuitos.

\section{Discusión y conclusiones}

Las representaciones sociales de la democracia, como cualquier objeto social, pueden ser abordadas desde diferentes metodologías. En trabajos anteriores (Ruiz \& Coy, 2004; Pineda \& Ruiz, 2009), empleando la asociación libre de palabras y una adaptación de los esquemas cognitivos de base (ver Abric, 1993), la democracia se asocia con conceptos como igualdad, libertad, participación o justicia. Sin embargo, para el caso 


\section{Ciencia}

colombiano, la investigación de Pineda y Ruiz (2009), muestra que la democracia se vincula también con la corrupción.

En la presente investigación, la corrupción parece ser el aspecto que más caracteriza a la democracia no como algo intrínseco a ella, sino externo; pero que marca claramente la representación social de esta. Otros aspectos que la caracterizan son los relacionados con la elección de cargos y ciertos valores. A nivel factorial, cuatro dimensiones estructuran las representaciones sociales de la democracia, los valores asociados a ella, los mecanismos formales-constitucionales de elección de cargos, la democracia como mecanismo de mantenimiento de desigualdades sociales y la corrupción. El Índice de Valoración de laDemocracia muestra quelos aspectos negativos reciben unos promedios casi similares a los positivos y dentro de una distribución normal del indicador, existen diferencias regionales, departamentales, en el predominio de una visión más positiva o más negativa de la democracia.

A partir de estas dimensiones, correspondientes a los principios organizadores de las representaciones sociales (Doise, Clemence. \& Lorenzi-Cioldi, 1992), se identifican cuatro tipologías tentativas de sujetos. Tentativas en el sentido de que no se da el mismo nivel de variables intragrupo, de acuerdo con la estadística de Levene, pero si permiten apreciar que el posicionamiento ante la democracia es diverso, lo que permite identificar las representaciones sociales, en sentido literal del plural, sobre la democracia. Además, estas representaciones muestran su anclaje (Jodelet, 1989; Abric, 1993; Abric \& Campos, 1996; Guimelli, 1996) en variables demográficas, socioeconómicas (estrato, formación académica) $y$, también, de victimización.

En cuanto a estas experiencias de delitos, los resultados encontrados en el trabajo coinciden parcialmente con los de Rodríguez y Seligson (2009) y con la propuesta de Villarreal y Silva (2006) comentada anteriormente. Por un lado, para ciertos sujetos, una visión de poca identificación con la democracia se asocia con una mayor victimización de cierto tipo (daños coactivos, delincuencia en la calle); y de forma complementaria, una visión más positiva (idealista) la cual se encuentra vinculada con una menor victimización. Esto coincidiría con lo postulado por Villarreal y Silva, en el sentido de que una mayor experiencia como víctimas de delitos podría conducir a un menor apoyo a la democracia (y quizá lo inverso se tendría lugar: a menor victimización, mayor apoyo). También se halla que una mayor experiencia con las formas de victimización más violentas (es decir, muertes violentas o por accidentes, heridas, desapariciones) se relaciona con una valoración más positiva de la democracia y con una mayor identificación con sus aspectos formales (sistema de elecciones de cargos) tal como se evidencia en los reportes de Rodríguez y Seligson (2009). Estos últimos atribuyen la causa de esta relación al desarrollo de una empatía por 
los demás ciudadanos; a lo que se puede añadir que el apoyo a la democracia formal podría ser vista como la manera de buscar o garantizar que la voz de todos sea escuchada y que mediante eso se facilite la justicia efectiva respecto a las victimizaciones sufridas. Por último, cabe destacar que para algunos sujetos, los englobados en la categoría de «pesimistas» sus concepciones de la democracia no aparecen ligadas a las experiencias de victimización. Puesto que esta categoría se caracteriza por un estatus socioeconómico y nivel educativo mayor, el resultado para este grupo podría considerarse coincidente con el hallazgo en estudios previos de que los sujetos con mayor estrato socioeconómico consideraban menos central la democracia que los de estratos medios y bajos (Ruiz \& Coy, 2004).

Por último, las representaciones sociales suelen ser estudiadas como variables predictoras de otros constructos como las prácticas sociales. Aquí, en cambio, hemos analizado variables -de victimización- que podrían desempeñar un papel predictor sobre dichas representaciones. El recurso a análisis de varianza o correlaciones muestra que deben ser tomados en cuenta con precaución, ya que las relaciones lineales que muestran los coeficientes significativos pueden matizarse. Así, la proyección cartográfica del Índice de Valoración de la Democracia y uno de los indicadores de victimización (el que reúne las formas de victimización más frecuentes) muestra que esta relación no es lineal, al menos a nivel cartográfico. Ello es ex- plicable por que en cada contexto social pueden intervenir variables diversas y de forma diferencial que se asocien con las representaciones sociales de la democracia. Para el caso colombiano, las regiones del país presentan diferentes niveles de desarrollo económico, devenires históricos particulares desde la época de la independencia, e intensidades y formas específicas del conflicto armado que aqueja al país desde hace décadas, lo que se plasma en la exposición de Flórez (2007) acerca de la influencia de los grupos armados en las distintas regiones del país.

Muchas de estas variables no se han tomado en cuenta aquí, por la necesaria delimitación de objetivos y recursos que caracteriza a toda investigación. Futuros desarrollos investigativos han de dar cuenta de la generabilidad de estos resultados obtenidos en este caso en muestras no aleatorias, pero numerosas y equiparables entre departamentos (estudiantes del SENA y de unas pocas carreras universitarias). 


\section{Ciencia}

\section{Referencias}

Abric,J.C.(1993).Prátiquesetreprésentationssociales. París: PUF.

Abric, J.C. \& Campos, P. (1996). Les éducateurs et leur représentation sociale de l'enfant de rue au Brésil. En J. Abric (Coordinador). Exclusiónsociale,insertionetprévention. (137-150), Saint-Agné, Erès.

Acosta, Y. (2009). El conflicto político venezolano.Unalecturadesdelapsicologíasocial. XXXII Congreso Interamericano de Psicología, Ciudad de Guatemala.

Amnistía Internacional (1994). Violencia política en Colombia. Madrid: AI

Cárdenas, M., Parra, L., Picón, J., Pineda, H. \& Rojas, R. (2007). Las representaciones sociales de la política y la democracia. Última Década, 55-80.

Comisión Nacional para la Investigación sobre la Desaparición de Personas, CONADEP (1984). Informe Nunca más. Recuperado de http://www.enmanosdenadie.com. ar/wp-content/uploads/2013/03/nunca-mas-conadep.pdf.

Comisión Presidencial Asesora para la Calificación de Detenidos Desaparecidos, Ejecutados Políticos y Víctimas de Prisión Política y Tortura(2011).InformedelaComisión PresidencialAsesoraparalaCalificacióndeDe-
tenidosDesaparecidos,EjecutadosPolíticosy VíctimasdePrisiónPolíticayTortura.Recuperado de http://www.indh.cl/wp-content/ uploads/2011/10/Informe2011.pdf

Díaz, A. (2006). Representaciones sociales juveniles sobre la democracia. Anaquel, 13, 180-191.

Doise, W., Clémence, A. \& Lorenzi-Cioldi, F. (1992).Représentationssocialesetanalyses de données. Ginebra: PUG.

Doise, W., Spini, D. \& Clémence, A. (1999). Human rights studied as social representations in a cross-national context. European Journal of Social Psychology. 29 (1), 1-29.

Echebarría, A. \& González, J. (1995). Xenofobia: representación social del inmigrante y barreras intergrupales. En E. Garrido y C. Herrero(Comps.).PsicologíaPolítica,Jurídica y Ambiental. Madrid: Eudema. 51-63.

Echebarría, A. \& Alvarez, J. (1996). Representaciones sociales de la democracia y el sistema electoral: un estudio comparativo entre México y el País Vasco. Revista de Psicología Social, 11(1), 47-69.

Flórez,J.A.(2007).La participaciónelectoralatípicacomovariablederiesgo.Recuperadode http://www.terra.com.co/elecciones_2007/ articulo/html/vbe530.htm

Flores, A. (1999). La Tradición Autoritaria: Violencia y Democracia en elPerú. Biblioteca Virtual de Ciencias Sociales. Recuperado 
de http://www.cholonautas.edu.pe/modulo/upload/Flores\%20Galindo.pdf.

Grupo de Memoria Histórica de la Comisión Nacional de Reparación y Reconciliación (2013). ¡Basta ya! Colombia; Memorias de guerra y dignidad. Bogotá: Imprenta Nacional.

Guimelli, C. (1996). La déviance vue par les instances chargées du maintien de l'ordre. En J. Abric (Coordinador). Exclusión sociale, insertion et prévention. (125-136), Saint-Agné, Erès.

Goodhart, M. (2007). Deliberative global politics: discourse and democracy in a divided World. Swiss Political Science Review, 13 (4), 681-90.

Hernández,A.(1986).LaseleccionesenColombia: análisisjurídico-político.Memorias delColoquio Iberoamericano de Derecho Electoral. México: Cuadernos del CAPEL, 18-23.

International Committee of the Red Cross (2000). CountryReportElSalvadorICRCWorldwideConsultation ontheRulesofWar.Recuperado de http://www.greenbergresearch. com/articles/1927/3135_salvador.pdf.

Comisión Presidencial Asesora para la Calificación de Detenidos Desaparecidos, Ejecutados Políticos y Víctimas de Prisión Política y Tortura(2011).InformedelaComisiónPresidencialAsesoraparalaCalificacióndeDetenidosDesaparecidos,EjecutadosPolíticosy VíctimasdePrisiónPolíticayTortura.Recu- perado de http://www.indh.cl/wp-content/ uploads/2011/10/Informe2011.pdf.

Jodelet,D.(1989).Lesreprésentationssociales.Paris: PUF.

Martín, Q., Cabero, Ma. T. \& De Paz, Y.R. (2007). TratamientoestadísticodedatoscomSPSS. Madrid: Thomson.

Méndez, J., O’Donnell, G. \& Pinheiro, P.S. (2002). La (in)efectividad de la ley y La exclusión en América Latina. Buenos Aires: Paídós.

Naranjo,V.(2006).Representacionessocialessobreelgolpemilitarydictadura.Santiagode Chile: CEME - Archivo Chile.

Naredo, M. (2001). Seguridad urbana y miedo al crimen. Polis: Revista Académica 1(2).

PinedaA.\&Ruiz,J.I.(2009). Representacionessocialesdelademocracia:unestúdiocomparativo. XXXII Congreso Interamericano de Psicología, Ciudad de Guatemala.

Rodríguez, J. \& Seligson, M. (2009). Cultura PolíticadelademocraciaenColombia.Bogotá: América Barometer.

Ruiz, J. \& Coy, A. (2004). Esquemas cognitivos de base, contenido semântico y estructura de las representaciones sociales de la democracia.ActaColombianadePsicología.12,5-17.

Ruiz, J. \& Turcios, L. (2009). Percepción de seguridad, victimización y cultura ciudadana: sus relaciones en cinco contextos ibe- 


\section{Ciencia}

roamericanos. Pensamiento Psicológico, 6(13), 193-202.

Ruiz, J.I. (en revisión). Criminología Ambiental: unaperspectivamultinivel.Paraunlibroa editar por la Benemérita Universidad Autónoma de Puebla.

Schultze-Kraft,M.(2005). Pacificaciónypodercivil en Centroamérica. Bogotá: Norma.

Suárez, M. (1990). Speaking on unspeakable Toward a Psychosocial Understanding of Responses to Terror. Ethos. Journal of the SocietyforPsychological Anthropology, 18(3), 353-383.
Vázquez, V. \& García, T. (2009). Construcción socialdelademocraciaylafamiliademocrática. XXXII Congreso Interamericano de Psicología, Ciudad de Guatemala.

Villareal, A. \& Silva, B. (2006). Social cohesión, criminal victimization and perceived risk of crime in Brazilian neighborhoods. Social Forces, 84 (3), 1725-1753.

Yépez,M.(s/f).Representacionessocialesdelademocraciaenestudiantesuniversitariosdela UNAM. México: AMIC. 